\title{
ПЕЧЬ КИПЯЩЕГО СЛОЯ ДЛЯ ОБЖИГА ЦИНКОВЫХ КОНЦЕНТРАТОВ КАК ОБЪЕКТ РЕГУЛИРОВАНИЯ
}

\author{
Ивакина Светлана Анатольевна', \\ svetlana-ivakina@mail.ru \\ Мунц Владимир Александрович', \\ v.a.munts@urfu.ru \\ 1 Уральский федеральный университет им. первого Президента России Б.Н. Ельцина, \\ Россия, 620002, г. Екатеринбург, ул. Мира, 19.
}

Актуальность исследования обусловлена непрерывным увеличением использования цинка в мире. Получение цинка - дорогостоящий процесс, одним из этапов которого является обжиг сульфидных цинковых концентратов (шихты) в печи кипящего слоя с использованием воздушного дутья, обогащенного кислородом. Поскольку гранулометрический состав шихты, ее влажность и состав непостоянны, температура кипящего слоя может изменяться и требует непрерывного контроля со стороны оператора. Огромная масса слоя определяет большую инерционность слоя, поэтому необходимо использование системы автоматического регулирования.

Цель исследования: оптимизация работы печи кипящего слоя для обжига сульфидных цинковых концентратов, в частности, введение автоматического регулирования температуры кипящего слоя во избежание колебания температуры в широких пределах. Значительное понижение температуры слоя приводит к ухудшению обжига концентрата, как следствие, уменьшается выход конечного продукта - цинка. При повышении температуры существенно возрастает опасность шлакования слоя из-за возможных его локальных перегревов. Для этого необходимо на основе изученной кинетики окисления цинкового концентрата в печи кипящего слоя получить зависимость температуры слоя от расхода шихты как расчетным, так и экспериментальным путем. Объект: печь кипящего слоя Челябинского цинкового завода для обжига сульфидного цинкового концентрата.

Методы: получение расчетной зависимости температуры кипящего слоя от расхода шихты на основе изученной кинетики окисления цинкового концентрата; экспериментальное изучение динамики изменения температуры слоя при изменении расхода шихты.

Результаты. Разработана математическая модель переходных процессов в печи кипящего слоя для обжига цинковых концентратов, позволяющая рассчитать изменение температуры слоя и концентрации горючих веществ в нем при изменении расхода загружаемой в печь шихты. Сопоставление расчетов С экспериментальными данными показали хорошую сходимость результатов. На основании полученных данных создана приближенная модель печи кипящего слоя в ПТК «Овация» и разработан алгоритм регулятора температуры кипящего слоя. Внедрение на ПАО «ЧЦЗ» регулятора температуры позволит без участия оператора поддерживать заданную температуру кипящего слоя, постоянство горючих в слое, исключить недожег шихты и снизить опасность шлакования слоя.

\section{Ключевые слова:}

Цинковый концентрат, кипящий слой, автоматическое регулирование, постоянная времени, константа скорости химического реагирования.

\section{Введение}

Сульфидный цинковый концентрат получают методом флотации из размолотой руды, размер частиц составляет менее 74 мкм $[1,2]$. В процессе погрузки-разгрузки, транспортировки образуются конгломераты из частиц концентрата, что делает материал полидисперсным с размерами частиц от нескольких микрометром до нескольких миллиметров [3]. При его обжиге в печи кипящего слоя происходит сепарация частиц по размеру. Мелкодисперсные частицы выносятся из слоя газовыми потоками, в дальнейшем частицы улавливаются в агрегатах очистки газов (котел-утилизатор, циклоны, электрофильтры). Более крупные частицы и часть мелкодисперсных частиц образуют кипящий слой. Обжиг цинкового концентрата происходит в кипящем слое при постоянном контакте частиц друг с другом и потоками газа [4-7]. Частицы, вынесенные из слоя газовыми потоками, окисляются в надслоевом пространстве печи и в газоходах. Здесь расстояние между частицами достаточно большое и частицы контактируют только с газовы- ми потоками (обжиг во взвешенном состоянии). Доля вынесенных частиц из слоя зависит от соотношения скоростей газовых потоков и физических свойств частиц (размер, плотность, форма). Для печей кипящего слоя Челябинского цинкового завода ориентировочно можно считать, что продукты обжига делятся в следующем соотношении: огарок (продукт из кипящего слоя) - (30-40) \%, циклонная пыль - (50-60) \%, пыль электрофильтров $\approx 5 \%$. Огарок в среднем находится в печи несколько часов при температурах от 900 до $980{ }^{\circ} \mathrm{C}$. Температура в надслоевом пространстве печи практически равна температуре кипящего слоя, но в дальнейшем снижается до $500{ }^{\circ} \mathrm{C}$ в циклонах, до $350{ }^{\circ} \mathrm{C}$ в электрофильтрах.

Кипящий слой как объект регулирования обладает специфическими свойствами и требует введения такого дополнительного (по сравнению с традиционными методами сжигания) параметра регулирования, как температура кипящего слоя [8-10]. Значительное понижение температуры слоя приводит к ухудшению обжига концентрата, 
как следствие, уменьшается выход конечного продукта - цинка. При температуре менее $850{ }^{\circ} \mathrm{C}$ скорость окисления шихты становится недостаточной для поддержания горения, что может привести к потуханию и прекращению кипения. При температуре более $950{ }^{\circ} \mathrm{C}$ существенно возрастает опасность шлакования слоя из-за возможных его локальных перегревов.

Поскольку гранулометрический состав шихты, ее влажность и состав непостоянны, то температура кипящего слоя может изменяться и требует непрерывного контроля со стороны оператора [11]. На ПАО «ЧЦЗ» регулирование температуры слоя осуществляется путем изменения оператором частоты вращения электродвигателя ленточного транспортера (косвенный показатель количества загружаемой шихты в печь), без изменения количества подаваемого кислорода в печь. Огромная масса слоя определяет большую инерционность слоя, что требует использования системы автоматического регулирования [12-15]. Для ее внедрения необходимо на основе изученной кинетики окисления цинкового концентрата в печи кипящего слоя получить зависимость температуры слоя от расхода шихты как расчетным, так и экспериментальным путем, разработать алгоритм регулятора температуры и приближенную модель кипящего слоя.

\section{Экспериментальное определение динамики изменения температуры слоя при изменении расхода шихты}

Для управления технологическим процессом обжига сульфидных цинковых концентратов в печах кипящего слоя ПАО «ЧЦЗ» используется комплекс первичных и вторичных датчиков, объединенных в рабочую станцию на базе персонального компьютера. Система позволяет выводить на монитор ПК данные по температуре в различных точках печи и газового тракта, данные по расходу воздуха и кислорода, подаваемых в печь, давление (разрежение) в различных точках печи, индикацию работы оборудования (рис. 1).

Схема пода печи кипящего слоя представлена на рис. 2. На схеме обозначены: сектор А - форкамера, сектор В - сектор интенсивного окисления шихты, в секторах C установлены кессоны для охлаждения слоя, сектор D - сектор выгрузки огарка из печи. Номерами $1,3,4,5,6$ обозначены термопары, установленные на высоте 20-30 см от пода, выход термопары в печь на $5-10$ см от боковой стенки печи. Воздушное дутье, обогащенное кислородом до 25-35\%, подается в форкамеру печи и следующую за форкамерой половину печи (сектор В и половину сектора С в области расположения термопар 3 и 4). В оставшиеся сектора подается воздух. Кипящий слой с хорошей аэродинами-

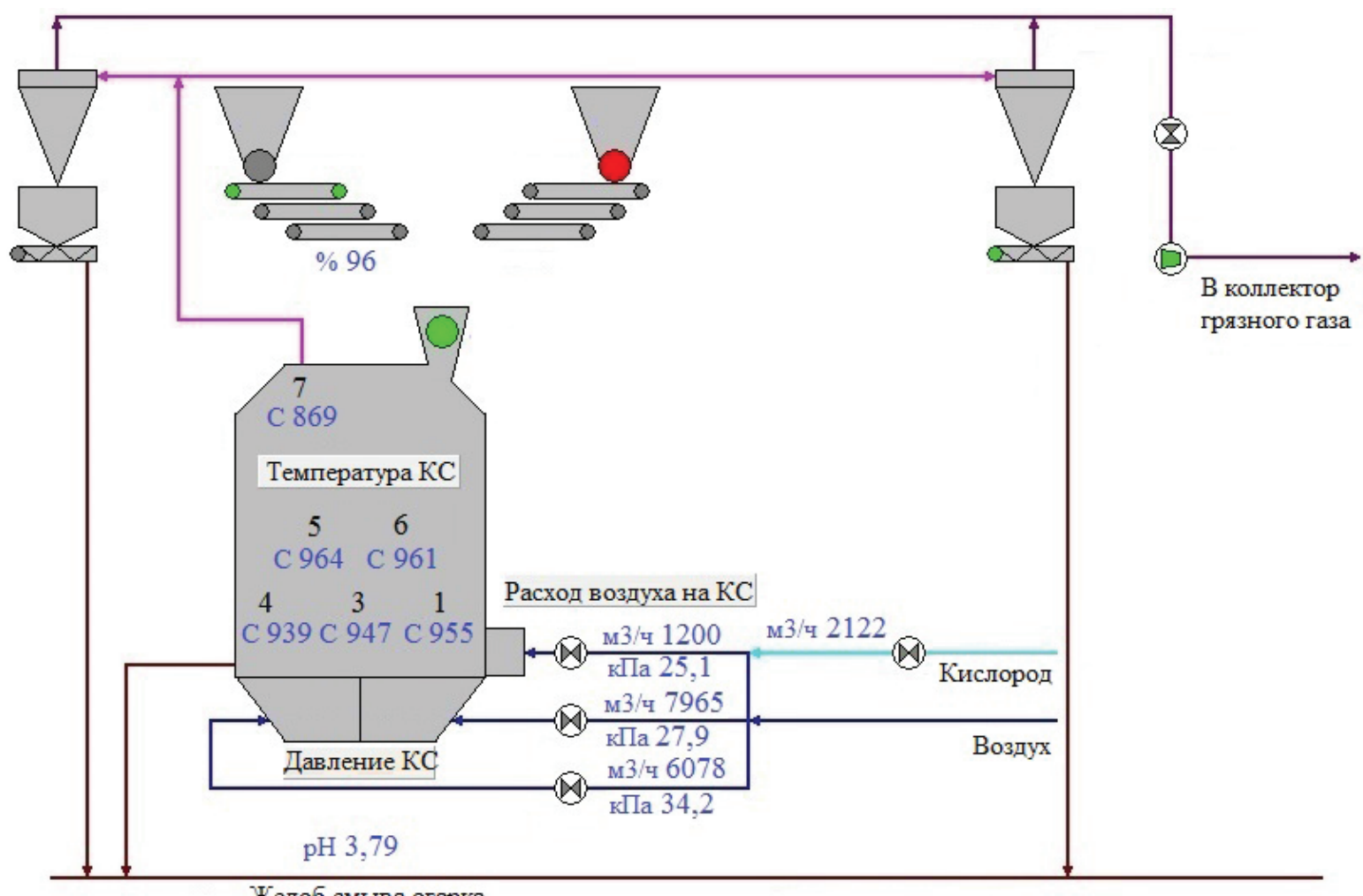

Желоб смыва огарка

Рис. 1. Видеограмма контроля и управления процессом обжига цинкового концентрата

Fig. 1. Videogram of monitoring and control of zinc concentrate roasting 
кой обладает высокой теплопроводностью, и разница температур в слое между точками 1 и 5 не превышает $35^{\circ} \mathrm{C}(3,5 \%)$, поэтому слой можно считать изотермичным (рис. 3 ).

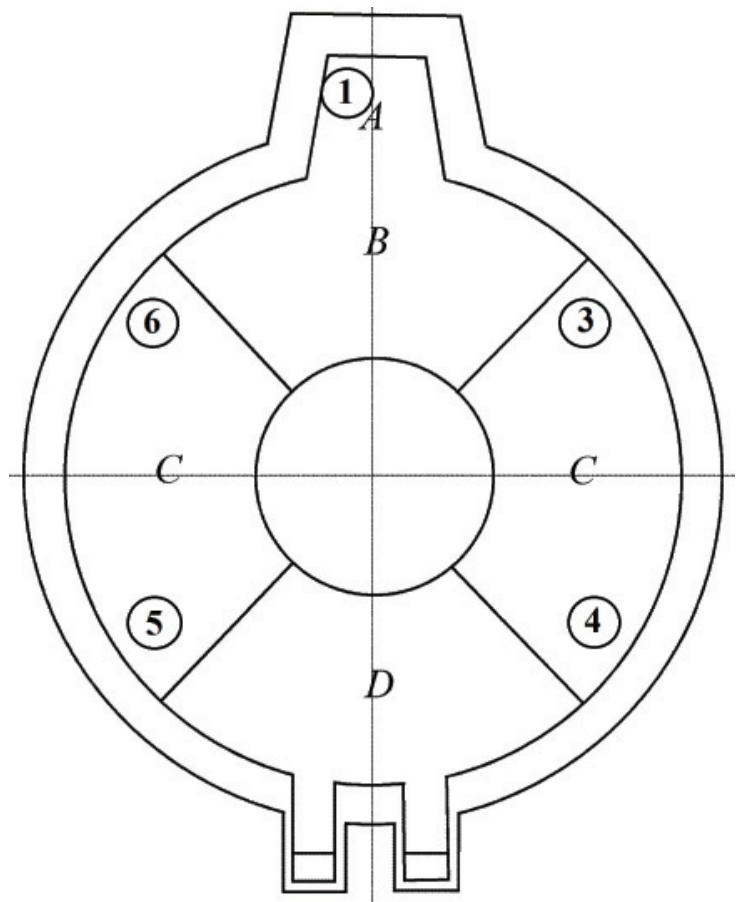

Рис. 2. Расположение термопар по поду печи

Fig. 2. Location of thermocouples by the furnace

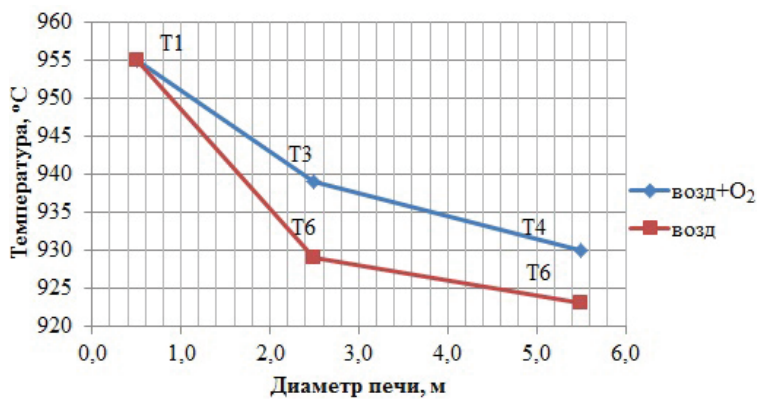

Рис. 3. Распределение температур в кипящем слое от зоны загрузки до зоны выгрузки шихты

Fig. 3. Distribution of temperatures in the fluidized bed from the loading zone to the discharge zone

Для определения взаимосвязи между частотой электропривода ленточного транспортера и температурой кипящего слоя построены графики изменения данных параметров в режиме реального времени в различных точках печи. Для анализа использовались данные за две смены по пяти термопарам трех печей. Для примера на рис. 4 приведены графики изменения температур и частоты вращения ленточного транспортера печи № 2 со 150 до 270 минут от начала смены.

Из графиков видно, что при ступенчатом возмущении (кратковременном уменьшении частоты электропривода ленточного транспортера) наблюдается изменение температуры с транспортным запаздыванием в 20 мин.
Для получения значения постоянной времени из эксперимента при импульсном возмущении необходимо преобразовать рис. 4 , достроив изменение, например, температуры в точке 3 до ступенчатого возмущения (рис. 5).

Определенная из данного эксперимента (рис. 5) постоянная времени составила 70,8 минут. На основании пяти экспериментальных значений постоянных времени (разных печей и смен) определено среднее значение, равное 68,7 минут.

\section{Моделирование переходных процессов} в кипящем слое

Для определения структуры и настройки регуляторов температуры кипящего слоя необходимо уметь рассчитывать характеристики слоя как объекта регулирования.

Для описания свойств кипящего слоя как объекта регулирования рассмотрена $[9,10]$ сопряженная система уравнений теплового и материального баланса. При моделировании использованы следующие предпосылки.

1. Перестройка полей концентраций газовых компонентов, обусловленная изменением концентрации горючих в его объеме, происходит практически мгновенно по сравнению со скоростью изменения температуры слоя. Это позволяет рассчитывать концентрации газов в каждый момент времени из решения стационарных уравнений горения, что значительно упрощает задачу.

2. Интенсивное перемешивание частиц кипящего слоя практически обеспечивает равномерное распределение горючих по объему и изотермичность слоя.

3. Реагирование шихты с кислородом протекает со скоростью реакции, определенной экспериментально. Константа скорости окисления шихты рассчитывается по выражению [16-19]:

$$
k S_{\text {шा }}=433 \exp \left(-\frac{14560}{8,314 T}\right), c^{-1},
$$

где $k$ - константа скорости химического реагирования шихты, отнесенная $\kappa$ внешней поверхности частицы, $\mathrm{M}^{3} /\left(\mathrm{M}^{2} \cdot \mathrm{c}\right) ; S_{\text {II }}$ - удельная внутренняя поверхность реагирования шихты, $\mathrm{m}^{2} / \mathrm{m}^{3}$.

4. Теплота, выделяющаяся за счет сгорания шихты, затрачивается на её подогрев до температуры слоя, испарение влаги, теплоотвод к поверхностям теплообмена и на подогрев продуктов сгорания до температуры слоя.

Введем безразмерную концентрацию горючих в слое как отношение массы горючих в слое $\kappa$ массе самого слоя:

$$
z=\frac{n \pi \delta^{3} \rho_{\mathrm{w}}}{6 V \rho_{\mathrm{c}}(1-\varepsilon)} .
$$

Здесь $z$ - безразмерная концентрация горючих в слое; $n$ - количество горючих частиц в слое; $\delta-$ диаметр горючих частиц, м; $\rho_{\text {ш }}, \rho_{\mathrm{c}}-$ плотность частиц шихты и слоя (огарка) соответственно, кг $/ \mathrm{M}^{3}$; $V$ - объем слоя, $\mathrm{M}^{3} ; \varepsilon$ - порозность слоя. 

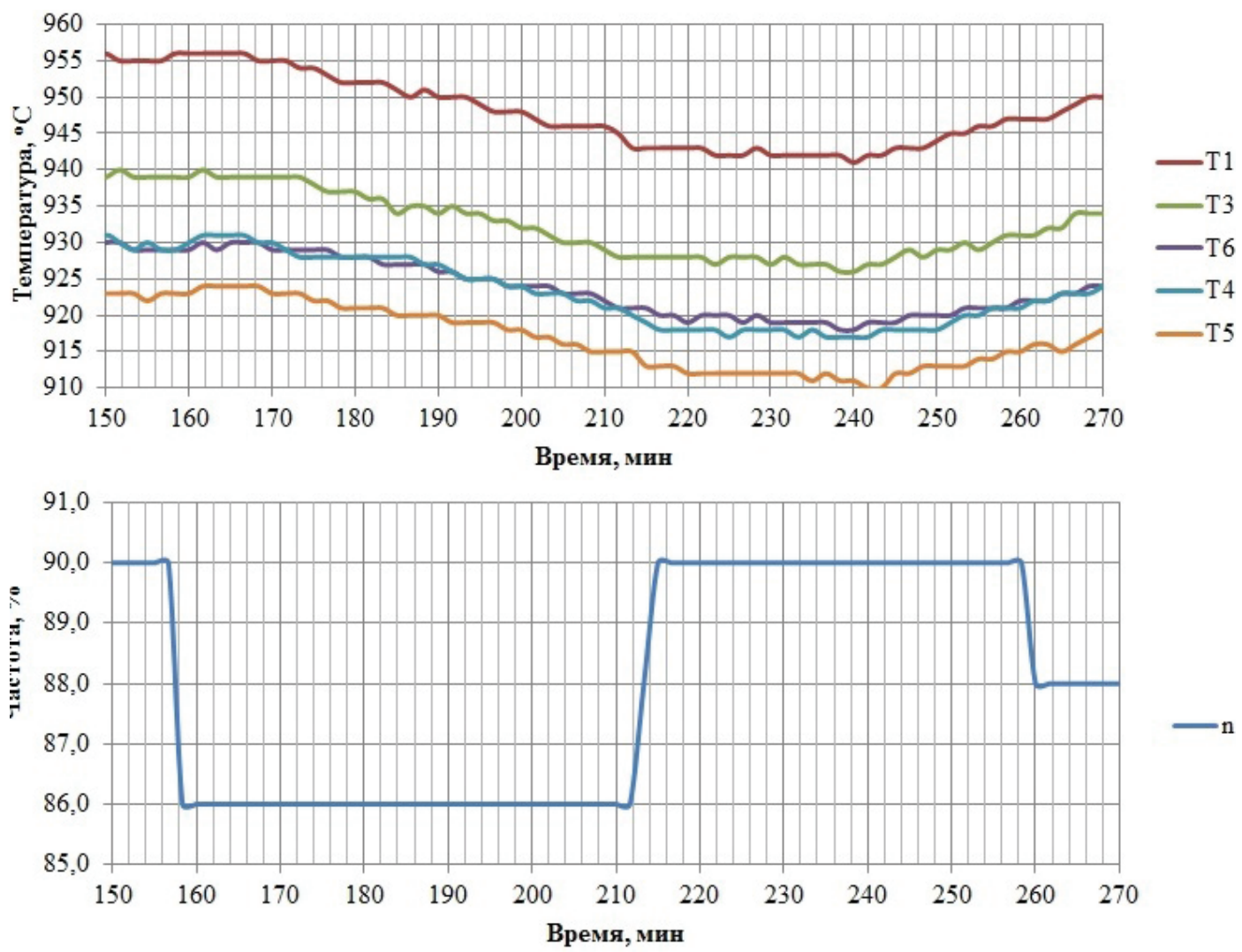

Рис. 4. Изменение температур в кипящем слое при изменении частоты вращения электропривода ленточного транспортера с течением времени

Fig. 4. Changing the bed temperatures with a change in the speed of the electric drive of the belt conveyor over time

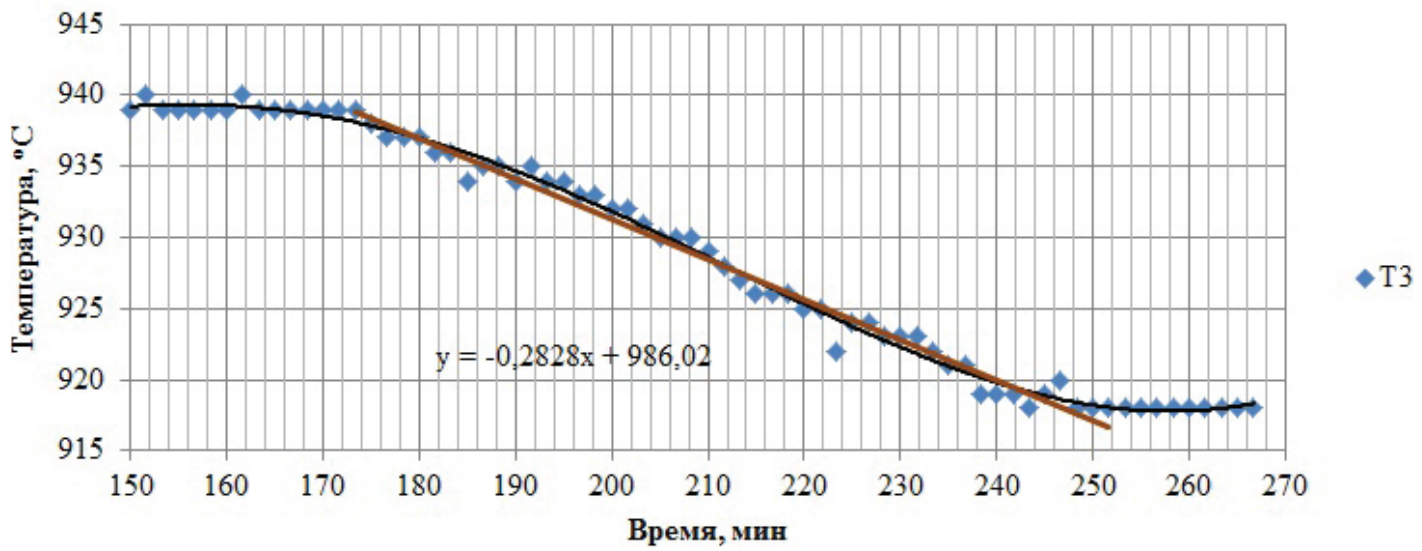

Pис. 5. Изменение температуры слоя Т3 при ступенчатом изменении частоты электропривода ленточного транспортера

Fig. 5. Changing the bed temperature T3 with a step change in the frequency drive belt conveyor

Внешняя удельная (отнесенная к единице объема слоя) поверхность реагирования горючих частиц определяется соотношением:

$$
S=\frac{n \pi \delta^{2}}{V}=\frac{6 \rho_{\mathrm{c}}(1-\varepsilon)}{\delta \rho_{\mathrm{m}}} \mathrm{z}=\frac{6}{\delta} \sigma z, \mathrm{M}^{2} / \mathrm{m}^{2},
$$

где $\sigma=\frac{\rho_{\mathrm{c}}(1-\varepsilon)}{\rho_{\text {шा }}}$.
Удельный поток реагирующей шихты равен:

$$
j=v_{\mathrm{mII}}\left(k S_{\mathrm{mI}} \zeta\right) S C_{\mathrm{O}_{2}}, \kappa \Gamma /\left(\mathrm{m}^{3} \cdot \mathrm{c}\right) .
$$

Здесь $\zeta=\frac{\delta}{6}$ - глубина проникновения реак-

ции, м; $v_{\text {ш }}$ - стехиометрический коэффициент реакции окисления цинкового концентрата (для шихты Челябинского цинкового завода $v_{\text {ш }}=1,91$ 
[16]); $C_{0_{2}}-$ концентрация кислорода в дутье, $\kappa \Gamma / \mathrm{M}^{3}$.

Подставляя (1) в (2) и вводя туда выражения для глубины проникновения реакции, получим выражение для удельного потока реагирующей шихты:

$$
\begin{gathered}
j=v_{\mathrm{II}}\left(k S_{\mathrm{\amalg I}}\right) \frac{\delta}{6} \frac{6}{\delta} \frac{\rho_{\mathrm{c}}(1-\varepsilon)}{\rho_{\mathrm{I}}} z C_{\mathrm{O}_{2}}= \\
=v_{\mathrm{II}}\left(k S_{\mathrm{II}}\right) \sigma z C_{\mathrm{O}_{2}}=j^{*} \mathrm{z}, \kappa \Gamma /\left(\mathrm{M}^{3} \cdot \mathrm{c}\right),
\end{gathered}
$$

где $j^{*}$ - удельный поток реагирующей шихты, отнесенный к концентрации горючих веществ, равной $1, \mathrm{\kappa} \Gamma /\left(\mathrm{M}^{3} \cdot \mathrm{c}\right)$.

При указанных предположениях система нестационарных уравнений для описания переходного процесса в печи кипящего слоя будет иметь следующий вид:

$$
\begin{gathered}
M c_{\mathrm{c}} \frac{d t_{\mathrm{c}}}{d \tau}=V j^{*} z Q_{\mathrm{H}}^{\mathrm{p}}-B\left(1-\frac{W^{ð}}{100}\right) c_{\mathrm{I}}\left(t_{\mathrm{c}}-t_{\mathrm{m}}\right)- \\
-B \frac{W^{ð}}{100} \chi-W F_{\mathrm{c}} c_{\mathrm{\Gamma}}\left(t_{\mathrm{c}}-t_{\mathrm{B}}\right)-k_{\mathrm{II}} F_{\mathrm{II}}\left(t_{\mathrm{c}}-t_{\mathrm{II}}\right), \\
V \rho_{\mathrm{c}}(1-\varepsilon) \frac{d z}{d \tau}=B\left(1-\frac{W^{\partial}}{100}-\frac{A^{\delta}}{100}\right)-V j^{*} z .
\end{gathered}
$$

Здесь $M$ - масса кипящего слоя, кг; $c_{c}, c_{\text {ш }}-$ теплоемкость слоя и шихты соответственно, кДж/(кг.К); $c_{\text {г }}$ - теплоемкость уходящих газов, кДж/( $\left.\mathrm{m}^{3} \cdot \kappa\right) ; t_{\mathrm{c}}$ - температура слоя, ${ }^{\circ} \mathrm{C} ; t_{\mathrm{m}}, t_{\mathrm{B}}, t_{\text {I }}-$ начальная температура шихты, воздуха и поверхностей нагрева соответственно, ${ }^{\circ} \mathrm{C} ; \tau-$ время, $\mathrm{c} ; Q_{\mathrm{H}}^{\mathrm{P}}-$ низшая рабочая теплота сгорания шихты, кДж/кг; $B$ - расход шихты, кг/с; $W^{\mathrm{p}}-$ влажность шихты, $\% ; \chi$ - теплота парообразования, кДж/кг; $w$ - скорость воздушного дутья, м/с; $F_{c}, F_{\text {п }}$ площадь сечения топки и площадь поверхностей теплообмена соответственно, $\mathbf{m}^{2} ; k_{\text {п }}$ - коэффициент теплопередачи от слоя к поверхностям теплообмена, кВт $/\left(\mathrm{M}^{2} \cdot \mathrm{K}\right) ; A^{\mathrm{p}}$ - зольность шихты в рабочем состоянии, \% .

Масса и объем кипящего слоя, входящие в уравнения (3) и (4), связаны с площадью поперечного

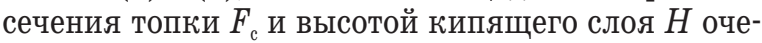
видным соотношением:

$$
M=H F_{\mathrm{c}}(1-\varepsilon) \rho_{\mathrm{c}} .
$$

Упростим уравнения (3) и (4), чтобы получить выражение, позволяющее пусть грубо, но наглядно представить влияние основных параметров на переходный процесс. Горение частиц шихты и испарение влаги будем рассматривать как один процесс, характеризуемый ее низшей теплотой сгорания. Пренебрежем теплотой, вносимой в топку воздухом, теплоносителем, поступающим в охлаждающие кессоны, и теплотой, затраченной на нагрев частиц, поступающих в слой. В этом случае уравнения преобразуются к виду:

$$
\begin{gathered}
M c_{\mathrm{c}} \frac{d t_{\mathrm{c}}}{d \tau}=V j^{*} z Q_{\mathrm{H}}^{\mathrm{p}}-w F_{\mathrm{c}} \mathrm{c}_{\mathrm{r}} t_{\mathrm{c}}-k_{\mathrm{I}} F_{\mathrm{I}} t_{\mathrm{c}}, \\
M \frac{d z}{d \tau}=B-V j^{*} \mathrm{z} .
\end{gathered}
$$

Выразив из уравнения (6) концентрацию горючих веществ в слое как

$$
z=\frac{B}{V j^{*}}-\frac{M}{V j} \frac{d z}{d \tau}
$$

и подставив ее в уравнение (5), получим уравнение теплового баланса в виде, удобном для качественного анализа:

$$
M c_{\mathrm{c}} \frac{d t_{\mathrm{c}}}{d \tau}=Q_{\mathrm{H}}^{\mathrm{p}}\left(B-M \frac{d z}{d \tau}\right)-w F_{\mathrm{c}} c_{\mathrm{r}} t_{\mathrm{c}}-k_{\mathrm{\Pi}} F_{\mathrm{\Pi}} t_{\mathrm{c}},
$$

Смысл уравнения (7) в следующем. В стационарном режиме $\frac{d t_{\mathrm{c}}}{d \tau}=\frac{d z}{d \tau}=0$ вся теплота $B Q_{\mathrm{H}}^{\mathrm{p}}$ затрачивается на нагрев продуктов сгорания и передается охлаждающим поверхностям. В переходных режимах (например, при уменьшении расхода топлива) сгорает не только поступающее топливо, но и часть горючих, аккумулированных в слое (при этом $\frac{d z}{d \tau}<0$ ). При увеличении расхода топлива горючие, наоборот, накапливаются в слое $\left(\frac{d z}{d \tau}>0\right)$, т. е. в слое сгорает меньше топлива, чем его туда подается. Если производная $\frac{d z}{d \tau}$ велика, т. е. величина $V \rho_{\text {c }} \frac{d z}{d \tau}$ сравнима с $B$, то влияние изменения концентрации горючих веществ в слое на изменение температуры слоя может быть существенным. Это происходит при достаточно высоких значениях $z$.

Для оценки постоянной времени кипящего слоя $[8,20,21]$ продифференцируем уравнение (5) по времени:

$$
M c_{\mathrm{c}} \frac{d^{2} t_{\mathrm{c}}}{d \tau^{2}}=V j^{*} z Q_{\mathrm{H}}^{\mathrm{p}} \frac{d z}{d \tau}-\left(w F_{\mathrm{c}} \mathrm{c}_{\mathrm{r}}+k_{\mathrm{H}} F_{\mathrm{I}}\right) \frac{d t_{\mathrm{c}}}{d \tau} .
$$

Считая поток $j^{*}$ постоянным, выразим из уравнения (7) производную $\frac{d z}{d \tau}$ :

$$
\frac{d z}{d \tau}=\frac{1}{M Q_{\mathrm{H}}^{\mathrm{p}}}\left[Q_{\mathrm{H}}^{\mathrm{p}} B-M c_{\mathrm{c}} \frac{d t_{\mathrm{c}}}{d \tau}-\left(w F_{\mathrm{c}} \mathrm{c}_{\mathrm{r}}+k_{\mathrm{H}} F_{\mathrm{I}}\right) d t_{\mathrm{c}}\right] .
$$

Подставив ее в (8), получим уравнение второго порядка для изменения температуры кипящего слоя в виде:

$$
\begin{gathered}
\frac{M c_{\mathrm{c}} \rho_{\mathrm{c}}(1-\varepsilon)}{j^{*}} \frac{d^{2} t_{\mathrm{c}}}{d \tau^{2}}+ \\
+\left[M c_{\mathrm{c}}+\frac{\rho_{\mathrm{c}}(1-\varepsilon)}{j^{*}}\left(w F_{\mathrm{c}} c_{\tilde{\mathrm{a}}}+k_{\mathrm{m}} F_{\mathrm{I}}\right)\right] \frac{d t_{\mathrm{c}}}{d \tau}= \\
=Q_{\mathrm{H}}^{\mathrm{p}} B-\left(w F_{\mathrm{c}} c_{\tilde{\mathrm{a}}}+k_{\mathrm{II}} F_{\mathrm{II}}\right) t_{\mathrm{c}} .
\end{gathered}
$$

При ступенчатом изменении расхода топлива в момент времени $\tau=0$ с $B_{0}$ до $B$ температура возра- 
стает за продолжительный отрезок времени с $t_{0}$ до $t_{c}$. Учитывая, что до внесения возмущения в стационарном состоянии наблюдалось равенство тепловыделений и теплоотвода

$$
Q_{\mathrm{H}}^{\mathrm{p}} B_{0}-\left(w F_{\mathrm{c}} c_{\tilde{\mathrm{a}}}+k_{\mathrm{\Pi}} F_{\mathrm{\Pi}}\right) t_{0}=0,
$$

вычтем уравнение (10) из (9) и, введя традиционно используемые обозначения при описании свойств объектов автоматического регулирования $B-B_{0}=x$ и $t_{\mathrm{c}}-t_{0}=y$, получим уравнение многоемкостного статического объекта [8] (кипящего слоя) в виде:

$$
T_{1} T_{2} \frac{d^{2} y}{d \tau^{2}}+\left(T_{1}+T_{2}\right) \frac{d y}{d \tau}+y=K_{\mathrm{y}} x .
$$

В случае монотонного изменения параметров можно, пренебрегая второй производной по времени уравнения (11), представить кипящий слой как одноемкостный статический объект:

$$
\left(T_{1}+T_{2}\right) \frac{d y}{d \tau}+y=K_{\mathrm{y}} x,
$$

в котором постоянная времени включает в себя два характерных слагаемых:

$$
\begin{gathered}
T_{1}=\frac{M c_{\mathrm{c}}}{w F_{\mathrm{c}} C_{\mathrm{\Gamma}}+k_{\mathrm{II}} F_{\mathrm{II}}}=\frac{H(1-\varepsilon) \rho_{\mathrm{c}} c_{\mathrm{c}}}{w C_{\mathrm{\Gamma}}+k_{\mathrm{II}} F_{\mathrm{I}} / F_{\mathrm{c}}}, \\
T_{2}=\frac{\rho_{\mathrm{c}}(1-\varepsilon)}{j^{*}}=\frac{\rho_{\mathrm{c}}(1-\varepsilon)}{v_{\mathrm{II}}\left(k S_{\mathrm{mI}}\right) C_{\mathrm{O}_{2}}} \frac{\rho_{\mathrm{II}}}{\rho_{\mathrm{c}}(1-\varepsilon)}= \\
=\frac{\rho_{\mathrm{II}}}{v_{\mathrm{II}}\left(k S_{\mathrm{m}}\right) C_{\mathrm{O}_{2}}} .
\end{gathered}
$$

Первая составляющая постоянной времени (12) характеризует соотношение между затратами теплоты на нагрев слоя, с одной стороны, и на нагрев продуктов сгорания и теплоносителя в трубах с другой. Расчет второй составляющей постоянной времени по соотношению (13) затруднен хотя бы из-за того, что, как правило, удельная поверхность частиц в слое либо средний диаметр частиц и удельный поток выгорающего вещества с достаточной точностью не известны. Используя соотношение между расходом топлива и концентрацией горючих веществ в слое для начального стационарного состояния $B_{0}=V j z_{0}$, можно записать:

$$
T_{2}=\frac{\rho_{\mathrm{c}}(1-\varepsilon)}{j^{*}}=\frac{V \rho_{\mathrm{c}}(1-\varepsilon)}{B_{0}} z_{0}=\frac{M z_{0}}{B_{0}} .
$$

Используя данное выражение, вторую составляющую постоянной времени $T_{2}$ можно легко определить из эксперимента.

Таким образом, на инерционность кипящего слоя основное влияние оказывают его высота, условия выгорания (коэффициент избытка воздуха, скорость дутья) и концентрация горючих в слое, зависящая от кинетических характеристик реагирования шихты. Оценочные расчеты для обжиговой печи № 2 Челябинского цинкового завода дают следующие значения для постоянных времени. Так, $T_{1}=65$ минут, $T_{2}=3,5$ минуты, тогда суммарная постоянная времени составляет 68,5 минут. Таким образом, основная инерция заключается в чрезвычайно большой массе слоя (более 100 тонн материала). И если концентрация горючих меняется сравнительно быстро (в течение 15 минут) (рис. $6, a)$, то температура слоя при изменении расхода шихты выходит на новое стационарное состояние чрезвычайно медленно, в течение 3,5 часов (рис. 6, б).

На рис. 7 приведено сопоставление расчетных и экспериментальных данных на отрезке времени со 170 по 210 минуту от начала смены.

Величина коэффициента усиления может быть рассчитана как

$$
K_{\mathrm{y}}=\frac{Q_{\mathrm{H}}^{\mathrm{p}}}{w F_{\mathrm{c}} c_{\Gamma}+k_{\mathrm{H}} F_{\mathrm{I}}},(\kappa \mathrm{c}) / \kappa \Gamma .
$$

В физическом смысле коэффициент усиления представляет собой изменение температуры слоя при изменении расхода шихты на 1 кг/с.

Для решения системы уравнений (5) и (6) примем начальные условия:

Начальные условия:

$$
\tau=0, z_{0}=\frac{B_{0}}{V j}, t_{0}=\frac{Q_{\mathrm{H}}^{\mathrm{p}} B_{0}}{w F_{\mathrm{c}} C_{\mathrm{\Gamma}}+k_{\mathrm{I}} F_{\mathrm{II}}} .
$$

Приведем уравнения к безразмерному виду, для этого введем безразмерную температуру $\vartheta=\frac{t_{\mathrm{c}}}{t_{0}}$ и безразмерное время $\xi=\frac{B \tau}{V \rho_{c}(1-\varepsilon)}=\frac{B \tau}{M}$. Тогда система уравнений будет иметь следующий вид:

$$
\begin{gathered}
\sigma \frac{d \vartheta}{d \xi}=z-\psi \vartheta, \\
\frac{d z}{d \xi}=1-\varphi z .
\end{gathered}
$$

Здесь:

$$
\varphi=\frac{V j^{*}}{B}, \sigma=\frac{B c_{\mathrm{c}} t_{0}}{V j^{*} Q_{\mathrm{H}}^{\mathrm{p}}}, \psi=\frac{\left(w F_{\mathrm{c}} c_{\mathrm{r}}+k_{\mathrm{H}} F_{\mathrm{I}}\right) t_{0}}{V j^{*} Q_{\mathrm{H}}^{\mathrm{p}}} .
$$

Начальные условия:

$$
\xi=0, z_{0}=\frac{1}{\varphi}=\frac{B_{0}}{V j^{*}}, \vartheta_{0}=\frac{1}{\varphi \psi}=\frac{Q_{\mathrm{H}}^{\mathrm{p}} B_{0}}{\left(w F_{\mathrm{c}} c_{\mathrm{\Gamma}}+k_{\mathrm{I}} F_{\mathrm{I}}\right) t_{0}} .
$$

Решение системы уравнений имеет следующий вид:

$$
\begin{gathered}
\vartheta=\left(\vartheta_{0}+\frac{z_{0}-\sigma / \psi}{\sigma \varphi-\psi}\right) \exp \left(-\frac{\psi}{\sigma} \xi\right)+ \\
+\frac{\sigma / \psi-1 / \varphi}{\sigma \varphi-\psi}+\frac{1 / \varphi-z_{0}}{\sigma \varphi-\psi} \exp (-\varphi \xi), \\
z=\left(z_{0}-\frac{1}{\varphi}\right) \exp (-\varphi \xi)+\frac{1}{\varphi} .
\end{gathered}
$$



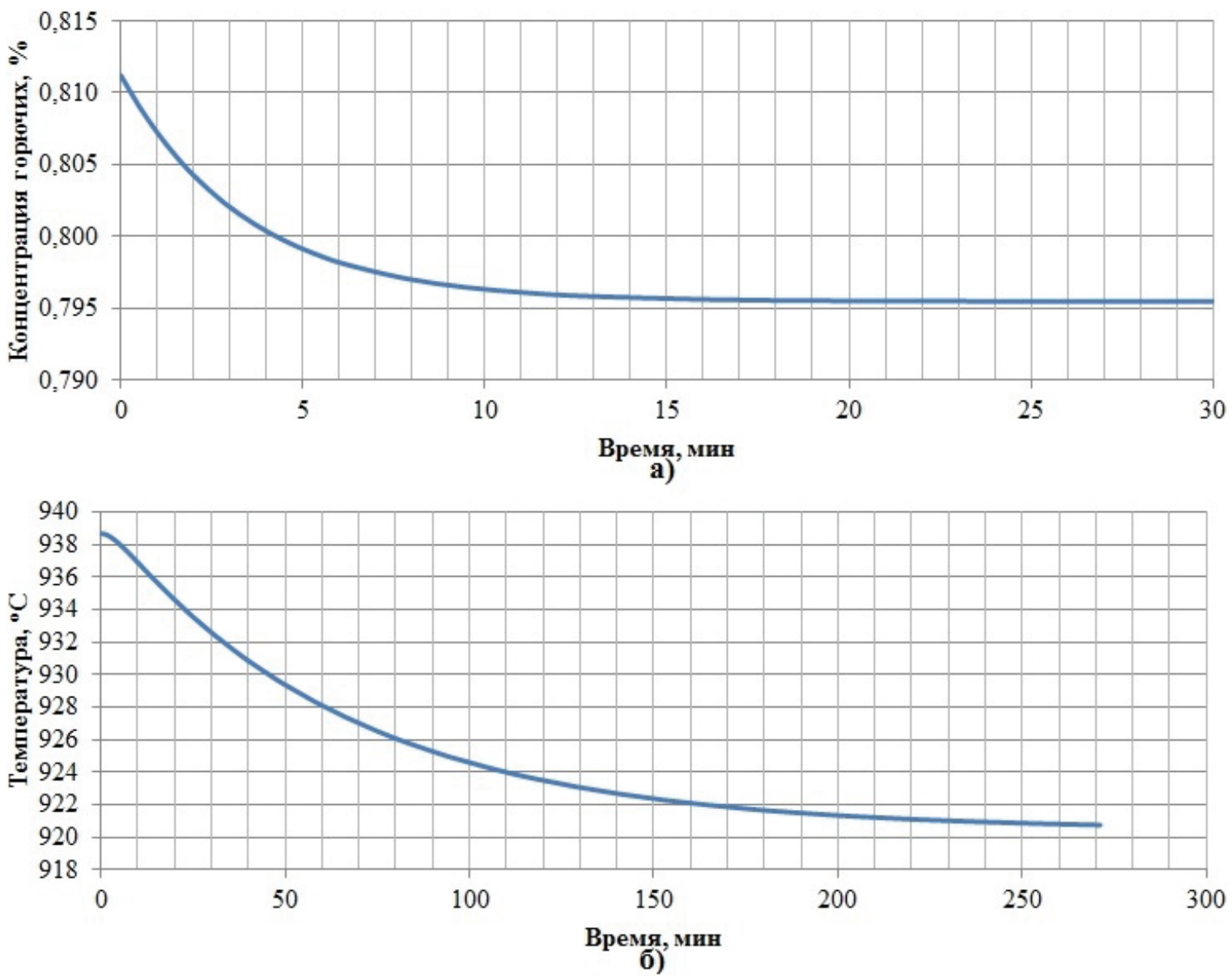

Pис. 6. а) изменение концентрации горючих с течением времени; б) изменение температуры слоя с течением времени

Fig. 6. a) changing the combustible concentration over time; b) changing the bed temperature over time

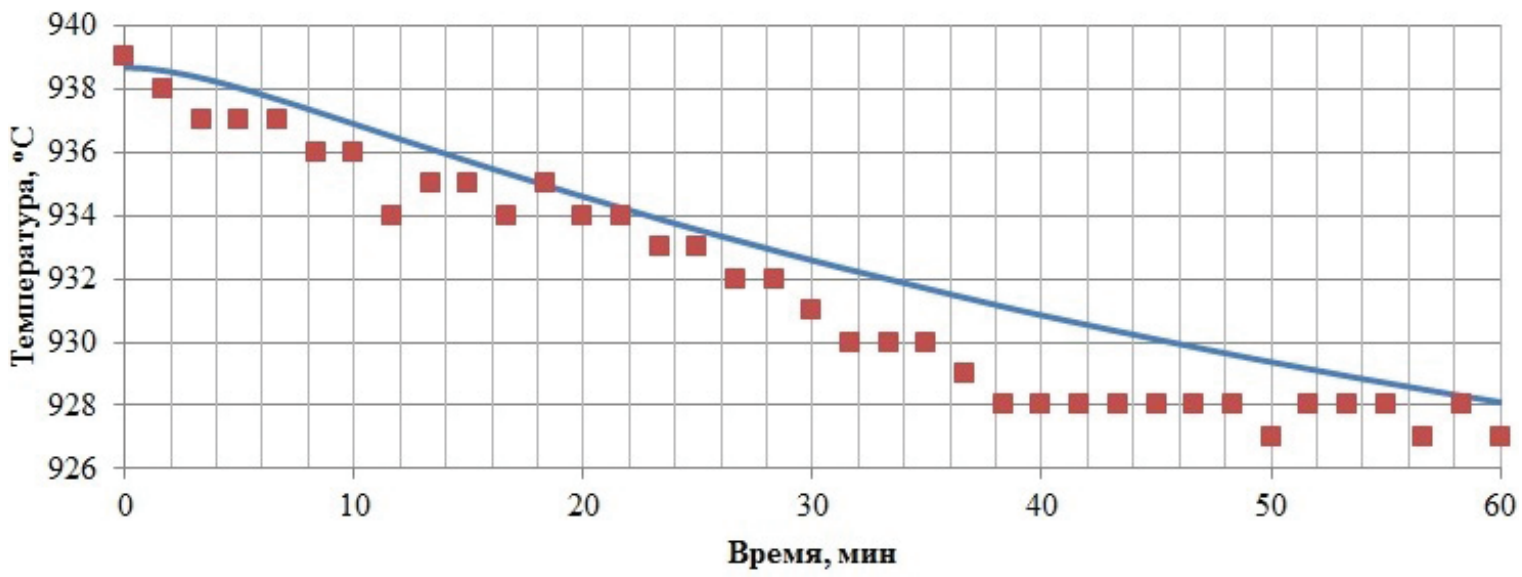

Рис. 7. Сопоставление расчетных и экспериментальных данных по изменению температуры слоя с течением времени при ступенчатом изменении расхода шихты: линия - расчет, точки - эксперимент

Fig. 7. Comparison of the calculated and experimental data on the bed temperature change over time with a step change in the charge rate: line - calculation, points - experiment

\section{Регулятор температуры кипящего слоя}

Регулятор предназначен для поддержания заданной температуры в печи кипящего слоя путем изменения частоты вращения ленточного транспортера.
Регулятор должен поддерживать на заданном уровне максимальную из температур кипящего слоя, приведенных на рис. 3. У оператора должна быть возможность выводить из расчета максимальной температуры любой из датчиков в случае, 
если их показания некорректны. При недостоверности показаний датчика его значение должно выводиться из расчета максимальной температуры автоматически.

Для определения оптимальных настроек регулятора на основании расчетных и экспериментальных данных создана приближенная модель печи кипящего слоя в ПТК «Овация» и разработан алгоритм регулирующего контура для поддержания температуры кипящего слоя на заданном значении, в основе которого лежит стандартный ПИ-регулятор [20, 21]. Структурная схема регулирующего контура приведена на рис. 8.

Модель кипящего слоя представляет собой последовательность типовых логических звеньев: звено ограничения скорости изменения частоты вращения ленточного транспортера (1 \% в минуту); звено транспортного запаздывания (20 мин); апериодическое звено, отвечающее за динамические характеристики объекта (постоянная времени $T=68,7$ мин); звено нелинейности, преобразующее процент частоты вращения ленточного транспортера в градусы температуры кипящего слоя. На рис. 9 представлены графики задающих воздействий регулирующего контура и переходный процесс в модели кипящего слоя.

До 11:00:00 система находилась в стационарном состоянии при температуре $940{ }^{\circ} \mathrm{C}$ с частотой вращения электропривода ленточного транспортера $87 \%$. В 11:00:00 нанесено ступенчатое возмущение регулятору изменением задания температуры на $920^{\circ} \mathrm{C}$. Регулятор в первый момент времени ступенчато изменяет задание по частоте вращения транспортера до 77 \% (величина изменения определяется величиной пропорциональной составляющей), постепенно продолжая снижать ее до момента начала изменения температуры (интегральная составляющая) (11:20:00). По истечении времени транспортного запаздывания (при появлении реакции регулируемого параметра на изменение частоты вращения) регулятор постепенно снимает возмущение (увеличивает частоту вращения) до выхода температуры на заданное значение (11:55:00). После этого происходит временный выход регулируемого значения температуры за заданное значение в пределах $2,5{ }^{\circ} \mathrm{C}$ (перерегулирование) и постепенный выход ее на стационарное значение. Выход температуры на заданное значение происходит в течение 55 минут с момента внесения возмущения в систему.

Настройки регулятора (коэффициент усиления $K_{\text {п }}=5$, постоянная времени интегрирования $T_{\text {и }}=1200 \mathrm{c}$ ) подобраны эмпирическим путем в созданной модели, исходя из следующих соображений [22]:

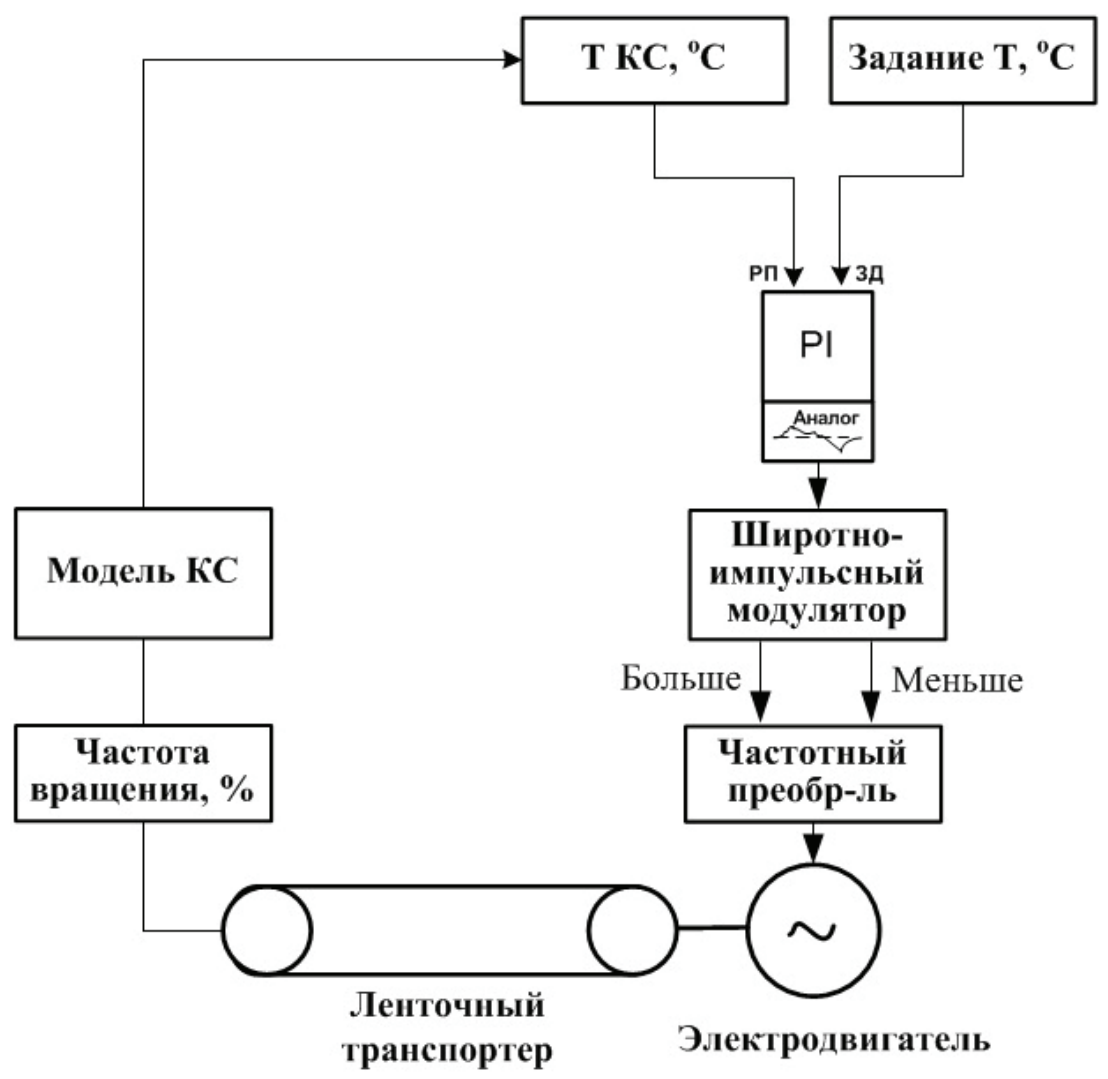

Рис. 8. Структурная схема регулятора температуры кипящего слоя

Fig. 8. Structural scheme of the fluidized bed temperature regulator 


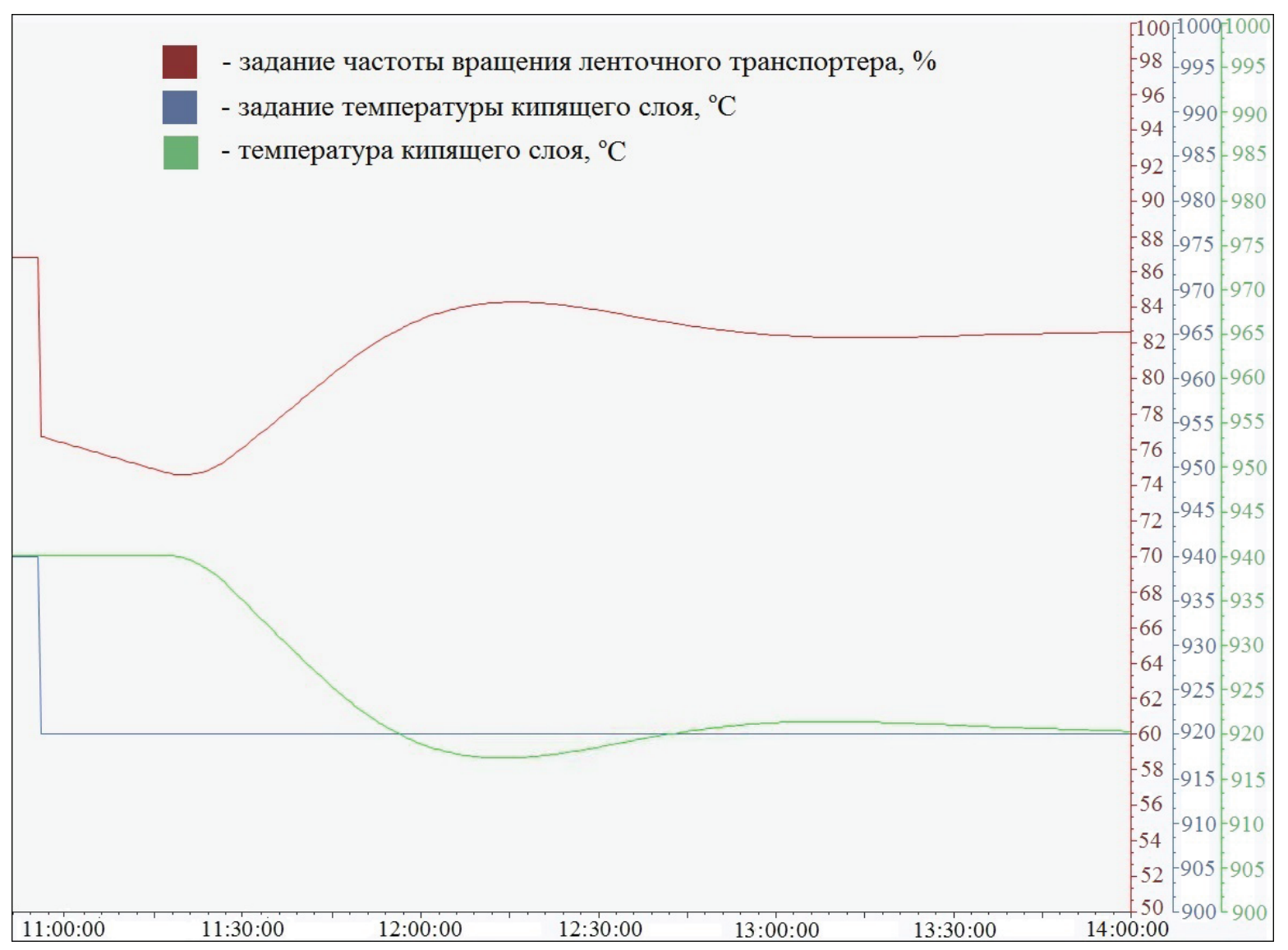

Рис. 9. Графики задающих воздействий регулирующего контура и переходный процесс в модели кипящего слоя

Fig. 9. Graphs of control actions of the control loop and the transient process in the fluidized bed model

1) увеличение пропорционального коэффициента $\left(K_{\text {п }}\right)$ увеличивает быстродействие регулятора, но снижает запас устойчивости;

2) увеличение постоянной времени интегрирования $\left(T_{\text {и }}\right)$ увеличивает запас устойчивости, но ошибка регулирования с течением времени уменьшается медленнее (выход на стационарное значение за более длительное время).

\section{Заключение}

Разработана математическая модель переходных процессов в печи кипящего слоя для обжига цинковых концентратов, позволяющая рассчитать изменение температуры слоя и концентрации го-

\section{СПИСОК ЛИТЕРАТУРЫ}

1. Процессы и аппараты цветной металлургии / С.С. Набойченко, Н.Г. Агеев, С.В. Карелов и др. / под общ. ред. С.С. Набойченко. - Екатеринбург: Изд-во Урал. ун-та, 2013. - 564 с.

2. Марченко Н.В., Вершинина Е.П., Гильденбрандт Э.М. Металлургия тяжелых цветных металлов. - Красноярск: ИПК СФУ, 2009. - 394 c.

3. Benlyamani M., Ajersch F. Agglomeration of particles during roasting of zinc sulfide concentrates // Metallurgical Transactions B. - 1986. - V. 17. - P. 647-656. DOI: 10.1007/BF02657127

4. Roasting of a sulfide polymetallic concentrate in a fluidized bed furnace / Y.A. Savinova, V.A. Popov, A.B. Portov, L.S. Tse- рючих веществ в нем при изменении расхода загружаемой в печь шихты. Сопоставление расчетов с экспериментальными данными показали хорошую сходимость результатов.

На основании полученных данных создана приближенная модель печи кипящего слоя в ПТК «Овация» и разработан алгоритм регулятора температуры кипящего слоя.

Внедрение на ПАО «ЧЦЗ» регулятора температуры позволит без участия оператора поддерживать заданную температуру кипящего слоя, постоянство горючих в слое, исключить недожег шихты и снизить опасность шлакования слоя.

mekhman // Russian Metallurgy (Metally). - 2014. - V. 2014. P. 351-357. DOI: 10.1134/S0036029514050115

5. Jarosz P., Malecki S. Kinetics of the fluidised oxidation of zinc sulphide concentrates with an addition of inert materials // Archives of metallurgy and materials. - 2014. - V. 59. P. 1367-1372.

6. Natesan K., Philbrook W.0. Oxidation kinetic studies of zinc sulfide in a fluidized bed reactor // Metallurgical and Materials Transactions. - 1970. - V. 1. - P. 1353-1360. D0I: $10.1007 / \mathrm{BF} 02900254$

7. Oxidation of zinc sulfide in a fluidized bed / Y. Fukunaka, T. Monta, Z. Asaki, Y. Kondo // Metallurgical Transactions B. 1976. - V. 7. - P. 307-314. 
8. Плетнев Г.П. Автоматическое регулирование и защита теплоэнергетических установок. 2-е изд., перераб. и доп. - М., Энергия, 1976. - 424 с.

9. Мунц В.А., Филипповский Н.Ф., Баскаков А.П. Топка с кипящим слоем как объект регулирования // Теплоэнергетика. 1998. - № 6. - C. 15-19.

10. Control of thermal processes in a fluidised bed combustor (FBC)/ V.A. Munts, N.F. Filippovskij, A.P. Baskakov, E.Yu. Pavliok, Bo. Leckner // Proc. of $14^{\text {th }}$ International Conference on FBC. Vancouver, May 1997. - V. 2. - P. 857-862.

11. Characterization of moisture distribution in a fluidized bed / M. Farkhondehkavaki, M. Soleimani, M. Latifi, F. Berruti, C. Briens, J. McMillan // Measurement. - 2014. - V. 47. P. $150-160$

12. Embedded Optimization Methods for Industrial Automatic Control / H.J. Ferreau, S. Almér, R. Verschueren, M. Diehl, D. Frick, A. Domahidi, J.L. Jerez, G. Stathopoulos, C. Jones // IFAC-Papers0nLine. - 2017. - V. 50. - P. 13194-13209.

13. Geldart D. Condition and control of fluid-bed process // Powder Technology. - 1973. - № 7. - P. 285-292.

14. Ротач В.Я. Теория автоматического управления. 5 -е изд., перераб. и доп. - М.: ИД МЭИ, 2008. - 396 с.

15. Бесекерский В.А., Попов Е.П. Теория систем автоматического управления. - СПб.: Профессия, 2003. - 752 с.

16. Мунц В.А., Ивакина С.А., Чойнзонов Д.Б. Изучение кинетики окисления сульфидного цинкового концентрата в печи кипящего слоя // Вестник ЮУрГУ. Серия «Энергетика». - 2017. T. 17. - № 3. - C. 34-42. DOI: 10.14529/power170304
17. Мунц В.А., Ивакина С.А., Терентьев В.М. Газообразование при обжиге цинкового концентрата в кипящем слое // Цветные металлы. - 2017. - № 2. - C. 40-45. DOI: $10.17580 /$ tsm.2017.02.06

18. Паньшин А.М., Козлов П.А., Терентьев В.М. Кинетика окисления сульфидных цинковых концентратов // Цветные металлы. - 2014. - № 2. - С. 34-37.

19. Sofekun 0.A., Doraiswamy L.K. High-temperature oxidation of zinc sulfide: Kinetic modeling under conditions of strict kinetic control // Industrial and Engineering Chemistry Research. 1996. - V. 35. - P. 3163-3170.

20. Исматходжаева С.К., Кузищин В.Ф. Повышение эффективности АСР тепловой нагрузки паровых котлов при сжигании топлива нескольких видов // Теплоэнергетика. - 2017. - № 5. C. 82-94. DOI: 10.1134/S0040363617050034

21. Кузищин В.Ф., Мерзликина Е.И., Ван Ва Хоанг. ПИД-регулятор с предиктором и алгоритмом автоматической настройки: исследование эффективности для тепловых объектов // Теплоэнергетика. - 2017. - № 9. - С. 80-90. D0I: $10.1134 / \mathrm{S} 0040363617090053$

22. Наладка средств автоматизации и автоматических систем регулирования. Справочное пособие. 2-е изд. перераб. и доп. / А.С. Клюев, А.Т. Лебедев, С.А. Клюев, А.Г. Товарнов / под ред. А.С. Клюева. - М.: Энергоатомиздат, 1989. - 368 с.

Поступила 10.11.2017 2.

\section{Информация об авторах}

Ивакина С.A., аспирант кафедры теплоэнергетики и теплотехники Уральского федерального университета им. первого Президента России Б.Н. Ельцина.

Муни B.A., доктор технических наук, профессор, заведующий кафедрой теплоэнергетики и теплотехники Уральского федерального университета им. первого Президента России Б.Н. Ельцина. 


\title{
FLUIDIZED BED FURNACE FOR ROASTING ZINC CONCENTRATES AS A CONTROL OBJECT
}

\author{
Svetlana A. Ivakina', \\ svetlana-ivakina@mail.ru \\ Vladimir A. Munts', \\ v.a.munts@urfu.ru \\ 1 Ural Federal University named after the first President of Russia B.N. Yeltsin, \\ 19, Mira street, Ekaterinburg, 620002, Russia.
}

The relevance of the research is caused by continuous increase in using zinc in the world. Obtaining of zinc is an expensive process, one of the stages of which is roasting of sulfide zinc concentrates (charge) in a fluidized bed furnace using air blast enriched with oxygen. Since the granulometric composition of the charge, its moisture content and composition are variable, the temperature of the fluidized bed can vary and requires continuous monitoring by the operator. A huge mass of material determines the large bed inertia, therefore, it is necessary to use an automatic control system.

The main aim of the research is to optimize the operation of the fluidized bed furnace for roasting sulphide zinc concentrates, in particular, to introduce automatic control of the fluidized bed temperature in order to avoid temperature fluctuations over a wide range. A significant decrease of the bed temperature leads to degradation of the concentrate roasting, as a consequence, the yield of the final product (zinc) decreases. As the temperature rises, the danger of bed slagging increases substantially due to the possible local overheating. To do this, it is necessary to obtain the dependence of the bed temperature on the charge rate, both by calculation and experimentally, on the basis of the studied kinetics of zinc concentrate oxidation in the fluidized bed furnace.

Object of research is the fluidized bed furnace of the Chelyabinsk Zinc Plant for roasting sulphide zinc concentrate.

Methods: obtaining the calculated dependence of the fluidized bed temperature on the charge rate based on the studied kinetics of zinc concentrate oxidation; an experimental study of the dynamics of the change in the bed temperature with a change in the charge rate. Results. The authors have developed the mathematical model of the transient processes in the fluidized bed furnace for calcining zinc concentrates, which makes it possible to calculate the change in the bed temperature and the combustible substances concentration in it with a change in the charge rate. Comparison of the calculations with the experimental data showed good convergence of the results. On the basis of the obtained data, an approximate model of the fluidized bed furnace in the Ovatsiya PTA and an algorithm of the fluidized bed temperature regulator were developed. The introduction of a temperature regulator at the Chelyabinsk Zinc Plant will enable automatically maintain the specified fluidized bed temperature, the constancy of the combustible in the bed, eliminate undesirable charges, and reduce the danger of slagging the layer.

Key words:

Zinc concentrate, fluidized bed, automatic control, time constant, reaction velocity constant.

\section{REFERENCES}

1. Naboychenko S.S., Ageev N.G., Karelov S.V., Mamyachenkov C.V., Sergeev V.A. Protsessy i apparaty tsvetnoy metallurgii [Processes and Apparatuses of Non-Ferrous Metallurgy]. Ed. by S.S. Naboychenko. Ekaterinburg, Ural University Publ., 2013. 564 p.

2. Marchenko N.V., Vershinina E.P., Gildenbrandt E.M. Metallurgiya tyazhelykh tsvetnykh metallov [Metallurgy of Heavy NonFerrous Metals]. Krasnoyarsk, IPK SFU Publ., 2009. 394 p.

3. Benlyamani M., Ajersch F. Agglomeration of particles during roasting of zinc sulfide concentrates. Metallurgical Transactions B, 1986, vol. 17, pp. 647-656. DOI: 10.1007/BF02657127

4. Savinova Y.A., Popov V.A., Portov A.B., Tsemekhman L.S. Roasting of a Sulfide Polymetallic Concentrate in a Fluidized Bed Furnace. Russian Metallurgy (Metally), 2014, vol. 2014, pp. 351-357. D0I: 10.1134/S0036029514050115

5. Jarosz P., Malecki S. Kinetics of the fluidized oxidation of zinc sulphide concentrates with an addition of inert materials. Archives of metallurgy and materials, 2014, vol. 59, pp. 1367-1372.

6. Natesan K., Philbrook W.0. Oxidation kinetic studies of zinc sulfide in a fluidized bed reactor. Metallurgical and Materials Transactions, 1970, vol. 1, pp. 1353-1360. DOI: 10.1007/BF02900254

7. Fukunaka Y., Monta T., Asaki Z., Kondo Y. Oxidation of zinc sulfide in a fluidized bed. Metallurgical Transactions B, 1976, vol. 7, pp. 307-314.

8. Pletnev G.P. Avtomaticheskoe regulirovanie i zashchita teploehnergeticheskih ustanovok. [Automatic control and protection of thermal power plants]. Moscow, Energiya Publ., 1976. 424 p.
9. Munts V.A., Filippovskii N.F., Baskakov A.P. Topka s kipyashchim sloem kak obekt regulirovaniya [The fluidized bed combustion as control object]. Teploenergetika, 1998, no. 6, pp. 15-19.

10. Munts V.A., Filippovskij N.F., Baskakov A.P., Pavliok E.Yu., Leckner Bo. Control of thermal processes in a fluidised bed combustor (FBC). Proc. of $14^{\text {th }}$ International Conference on FBC. Vancouver May 1997. Vol. 2, pp. 857-862.

11. Farkhondehkavaki M., Soleimani M., Latifi M., Berruti F., Briens C., McMillan J. Characterization of moisture distribution in a fluidized bed. Measurement, 2014, vol. 47, pp. 150-160.

12. Ferreau H.J., Almér S., Verschueren R., Diehl M., Frick D., Domahidi A., Jerez J.L., Stathopoulos G., Jones C. Embedded Optimization Methods for Industrial Automatic Control. IFAC-PapersOnLine, 2017, vol. 50, pp. 13194-13209.

13. Geldart D. Condition and control of fluid-bed process. Powder Technology, 1973, no. 7, pp. 285-292.

14. Rotach V.Ya. Teoriya avtomaticheskogo upravleniya [Theory of automatic control]. Moscow, MEI Publ. house, 2008. 396 p.

15. Besekerskii V.A., Popov E.P. Teoriya sistem avtomaticheskogo upravleniya [The theory of automatic control systems]. St. Petersburg, Professiya Publ., 2003. 752 p.

16. Munts V.A., Ivakina S.A., Choynzonov D.B. Study of Sulphide Zinc Concentrates 0xidation Kinetics in the Fluidized Bed Furnace. Bulletin of the South Ural State University. Ser. Power Engineering, 2017, vol. 17, no. 3, pp. 34-42. In Rus. DOI: $10.14529 /$ power170304 
17. Munts V.A., Ivakina S.A., Terentev V.M. Gas Generation during Zinc Concentrate Annealing. Tsuetnye Metally, 2017, no. 2, pp. 40-45. In Rus. DOI: 10.17580/tsm.2017.02.06

18. Panshin A.M., Kozlov P.A., Terentev V.M. Kinetics of Oxidation of Sulfide Zinc Concentrates. Tsuetnye Metally, 2014, no. 2, pp. 34-37. In Rus.

19. Sofekun 0.A., Doraiswamy L.K. High-temperature oxidation of zinc sulfide: Kinetic modeling under conditions of strict kinetic control. Industrial and Engineering Chemistry Research, 1996, vol. 35 , pp. 3163-3170.

20. Ismatkhodzhaeva S.K., Kuzishchin V.F. [Increase of the ACS efficiency of the thermal load of steam boilers during the combustion of several types of fuel]. Teploenergetika, 2017, no. 5, pp. 82-94. In Rus. DOI: 10.1134/S0040363617050034
21. Kuzishchin V.F., Merzlikina E.I., Van Va Khoang PID controller with predictor and automatic tuning algorithm: efficiency study for thermal objects]. Teploenergetika, 2017, no. 9, pp. 80-90. In Rus. DOI: 10.1134/S0040363617090053

22. Klyuev A.S., Lebedev A.T., Klyuev S.A., Tovarnov A.G. Naladka sredstv avtomatizatsii i avtomaticheskikh sistem regulirovaniya. Spravochnoe posobie [Adjustment of automation and automatic control systems. Tutorial]. Ed. by A.S. Klyuev. Moscow, Energoatomizdat Publ., 1989. $368 \mathrm{p}$.

Received: 10 November 2017.

\section{Information about the authors}

Svetlana A. Ivakina, postgraduate, Ural Federal University named after the first President of Russia B.N. Yeltsin.

Vladimir A. Munts, Dr. Sc., professor, Ural Federal University named after the first President of Russia B.N. Yeltsin. 